\title{
Which soft contact lens power is better for piggyback fitting in keratoconus?
}

\author{
Miguel Romero-Jiménez ${ }^{\mathrm{a}, *}$, Jacinto Santodomingo-Rubido ${ }^{\mathrm{b}}$, Patricia Flores-Rodríguez $^{\mathrm{c}}$, \\ Jose Manuel González-Meijóme ${ }^{\mathrm{d}}$ \\ a MGR Doctores, Madrid, Spain \\ ${ }^{\mathrm{b}}$ Menicon Co. Ltd. (Madrid Office), Majadahonda, Madrid, Spain \\ ${ }^{\mathrm{c}}$ Pontificia Universidad Católica de Valparaíso, Chile \\ d Clinical \& Experimental Optometry Research Lab, Center of Physics, University of Minho, Braga, Portugal
}

\section{A R T I C L E I N F O}

\section{Article history:}

Received 7 June 2012

Received in revised form

12 September 2012

Accepted 2 October 2012

\section{Keywords:}

Piggyback

Contact lens

Cornea

Keratoconus

\begin{abstract}
A B S T R A T
Purpose: To evaluate the ipact of differente soft contact lens power in the anterior corneal curvature and regulareity in subjects with keratoconus.

Methods: Nineteen subjects (30 eyes) with keratoconus were included in the study. Six corneal topographies were taken with Pentacam Eye System over the naked eye and successively with soft lens (Senofilcon A) powers of $-3.00,-1.50,0.00,+1.50$ and $+3.00 \mathrm{D}$. Corneal measurements of mean central keratometry (MCK), maximum tangential curvature (TK), maximum front elevation (MFE) and eccentricity (Ecc) at 6 and $8 \mathrm{~mm}$ diameters as well as anterior corneal surface high order aberrations (i.e. total RMS, spherical- and coma-like and secondary astigmatism) were evaluated.

Results: Negative- and plano-powered soft lenses flattened ( $p<0.05$ in all cases), whereas positivepowered lenses did not induce any significant changes ( $p>0.05$ in all cases) in MCK in comparison to the naked eye. The TK power decreased with negative lenses $(p<0.05$ in both cases) and increased with $+3.00 \mathrm{D}$ lenses $(p=0.03)$ in comparison to the naked eye. No statistically significant differences were found in MFE with any soft lens power in comparison to the naked eye ( $p>0.05$ in all cases). Corneal eccentricity increased at $8 \mathrm{~mm}$ diameter for all lens powers ( $p<0.05$ in all cases). No statistically differences were found in HOA RMS and spherical-like aberration (both $p>0.05$ ). Statistically differences were found in coma-like and secondary astigmatism (both $p<0.05$ ).

Conclusion: Negative-powered soft contact lenses provide a flatter anterior surface in comparison to positive-powered lenses in subjects with keratoconus and thus they might be more suitable for piggyback contact lens fitting.
\end{abstract}

(C) 2012 British Contact Lens Association. Published by Elsevier Ltd. All rights reserved.

\section{Introduction}

Keratoconus is a progressive, bilateral and asymmetric corneal disorder with an incidence of 1 per 2000 in the general population $[1,2]$. This corneal degeneration is characterized by localized corneal thinning which leads to protrusion of the thinned cornea, high myopia and irregular astigmatism, thus affecting visual quality. Corneal thinning normally occurs in the inferior-temporal as well as the central cornea [3]. Although the etiology of keratoconus remains unclear, it is suspected to develop as a combination of genetic and environmental factors [4]. Currently, a combination of corneal topographic findings (i.e. inferior corneal steepening and superior-inferior corneal curvature asymmetry) and clinical signs

\footnotetext{
* Corresponding author. Current address: MGR Doctores, Vallehermoso 32, Bajo A, Madrid, Spain. Tel.: +34 62 8782201; fax: +34 915919737.

E-mail address: mrjlentes@gmail.com (M. Romero-Jiménez).
}

(i.e. Fleischer's ring, Vogt's striae and corneal scarring) are commonly used to diagnose and monitor the disease [5].

New surgical procedures to treat keratoconus have emerged in the last decade, including corneal ring segments implantation [6] and collagen cross linking [7]. However, gas-permeable (GP) contact lenses still represent the most common and successful treatment option for early to moderate cases of keratoconus. Nevertheless, the use of these lenses might be associated to increased on-eye lens discomfort in comparison to soft contact lens wear ultimately leading to reduced wearing time and eventually contact lens wear discontinuation.

Piggyback lens systems were first described by Baldone in the early 1970s for improving contact lens comfort in keratoconus subjects fitted with GP lenses alone [8,9]. The technique consists in fitting a GP lens onto a soft lens (Fig. 1). The soft lens is used to improve comfort, GP lens centration and to protect the cone apex from potential corneal scarring induced by the GP lens [10]. Although piggybacking has been associated to corneal swelling and neovascularization [11], relatively recent studies have shown that 


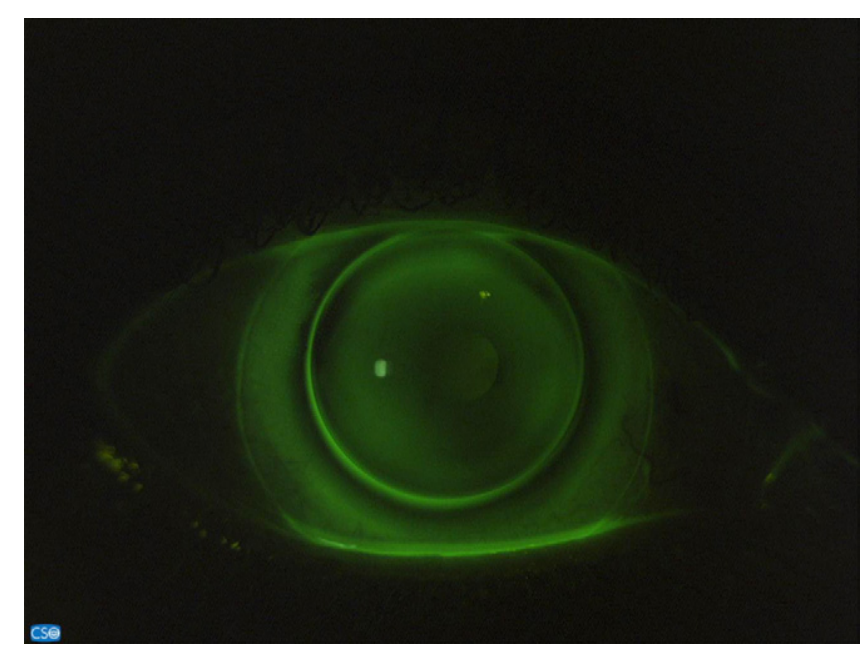

Fig. 1. On-eye piggyback lens fitting.

current high Dk GP lenses in combination with silicone hydrogel contact lenses provide sufficient oxygen transmissibility to avoid hypoxic-related complications during daily wear $[12,13]$. Current generations of silicone hydrogel lenses have a lower modulus of rigidity than earlier generations of silicone hydrogel and thus they are more likely to provide enhanced comfort for piggybacking with GP lenses.

Classically, the use of low positive-powered soft contact lens has been recommended for piggyback fittings as it is believed to create a lenticular bowl which facilitates GP lens centration, although the use of a negative-powered lens has been suggested perhaps more appropriate for fitting steep corneas (i.e. mean keratometry $>47.5 \mathrm{D}$ ) [14]. In fact, most studies report the use of positive-powered lenses in piggyback fittings [15,16]. In a pilot study on non-keratoconic eyes, O'Donnell and co-workers found positive-powered lenses facilitated spherical GP lens centration, despite negative-powered lenses generated a flatter anterior corneal surface for lens fitting [15]. As keratoconus is associated to corneal steppening in comparison to normal corneas, it might be expected that the use negative-powered soft lenses over the keratoconic cornea might generate a flatter surface onto which the GP lens might fits better. On the other hand, corneal eccentricity is normally increased in keratoconus in comparison to normal corneas [17]. Therefore, positive-powered soft lenses might reduce corneal eccentricity, thus facilitating the GP lens fitting. To the best of our knowledge, however, no previous study has evaluated which soft contact lens power provides a more regular anterior corneal surface to facilitate the fitting of a GP lens over the soft contact lens. Therefore, the purpose of this study is to evaluate how different soft lens powers affect anterior corneal surface in terms of central keratometry, corneal eccentricity and anterior corneal surface higher-order aberrations (HOA) in keratoconic eyes.

\section{Methods}

Subjects of at least 18 years of age diagnosed with keratoconus according to the keratoconus severity score were recruited for the study [4]. Full informed consent was obtained from all subjects prior to the start of all experimental work and data collection. The study followed the Tenets of the Declaration of Helsinki and was approved by the Institutional Review Board of MGR Doctores Ophthalmology Clinic.

In eligible subjects, comprehensive optometric and ophthalmologic examinations were performed in all subjects. The examination included Snellen uncorrected and corrected visual acuity tests (manifest refraction and contact lens), biomicroscopy examination, fundus evaluation, keratometry and corneal topographic analysis using the Pentacam Eye Scanner (Software version 1.16.r:23, Oculus Inc., Wetzlar, Germany). Eyes with a previous history of acute corneal hydrops, pellucid marginal degeneration, corneal surgery or any other ocular disease were excluded from the study. Corneal topography assessments were taken over the naked eye. Subsequently, a soft contact lens with a base curve of $8.40 \mathrm{~mm}$ and a power of $-3.00 \mathrm{D}$ was fitted, and new topography assessments were recorded with the lens over the cornea (Senofilcon, Acuvue Oasys, Johnson \& Johnson, Jacksonville, FL). The same process was repeated consecutively with lenses of $-1.50,0.00,+1.50$ and +3.00 D.

We analyzed mean central keratometry, maximum tangential curvature, maximum front elevation, and corneal eccentricity at 6 and $8 \mathrm{~mm}$ chord diameters. Additionally, HOA coefficients (3rd-6th order) and total HOA root mean square (RMS) were calculated for a $6.0 \mathrm{~mm}$ pupil. The corresponding RMS values were evaluated for the following types of optical aberrations: HOA up to the 27th term; spherical-like aberrations for 4th and 6th order Zernike terms; coma-like aberrations for 3rd and 5th order Zernike terms; and secondary astigmatism aberrations for 4th and 6th order Zernike terms.

\subsection{Statistical analysis}

Differences in mean keratometry and maximum tangential curvature for each of the contact lens powers in comparison to the naked eye were evaluated using the paired Student's $t$-test. Differences in maximum elevation, eccentricity and HOA values for each of the contact lens powers in comparison to the naked eye were evaluated using the Wilcoxon Singed Rank test. Statistical analyses were carried out using SPSS 15.0 software (SPSS Inc., Chicago, IL, USA). The level of statistical significance was taken as $5 \%$.

\section{Results}

Thirty eyes from 10 males (53\%) and 9 females (47\%) diagnosed with keratoconus were included in the study. The mean age $( \pm \mathrm{SD})$ was $26.4( \pm 6.2)$, ranging from 18 to 43 years. The mean flattest central keratometry was $48.10( \pm 4.84)$ and the mean steepest central keratometry was $51.67( \pm 5.39)$. According to the keratoconus severity score, the stage of keratoconus was mild in 20 eyes, moderate in 7 eyes and severe in 3 eyes. All subjects were current GP contact lens wearers.

Mean central keratometry flattened significantly with negativeand plano-powered soft lenses (all $p<0.05$ ), but did not change significantly with positive-powered lenses (all $p>0.05$ ). Maximum tangential curvature flattened significantly with negative-powered lenses (all $p<0.05$ ); did not change with the plano or $+1.50 \mathrm{D}$ lenses; and steepened significantly with $+3.00 \mathrm{D}$ lenses (Fig. 2). No statistically significant differences were found in maximum front elevation with any of the soft lens powers assessed in comparison to the naked eye (Table 1 ).

Similar corneal eccentricities at $6 \mathrm{~mm}$ chord diameters were found with the different contact lens powers assessed (all $p>0.05$ ). However, statistically significant differences were found between all soft contact powers in comparison to the naked eye at $8 \mathrm{~mm}$ chord diameter eccentricity. A trend for increasing corneal eccentricity with increasing lens power was found (Table 2).

No statistically significant differences were found between the different lens powers in HOA RMS, except for $-3.00 \mathrm{D}$ soft lens power $(p=0.012)$. No significant differences were found in spherical-like aberration for any of the lens powers (all $p>0.05)$. Significant differences were found in coma-like aberrations for all lens powers, except for the $+3.00 \mathrm{D}$ lens power 
Table 1

Mean central keratometry, tangential curvature and maximum front elevation.

\begin{tabular}{|c|c|c|c|c|c|c|}
\hline \multirow[t]{2}{*}{ Condition } & \multicolumn{2}{|c|}{ Mean central keratometry } & \multicolumn{2}{|c|}{ Tangential curvature } & \multicolumn{2}{|c|}{ Maximum front elevation } \\
\hline & Power (D) & $p^{*}$ & Power (D) & $p^{*}$ & Distance $(\mu \mathrm{m})$ & $p^{*}$ \\
\hline Naked-eye & \multicolumn{2}{|c|}{$49.95 \pm 5.03$} & \multicolumn{2}{|c|}{$55.72 \pm 7.19$} & \multicolumn{2}{|c|}{$36.21 \pm 22.20$} \\
\hline$-3.00 \mathrm{D}$ & $47.72 \pm 4.60$ & $<0.001$ & $53.70 \pm 6.56$ & $<0.001$ & $33.69 \pm 18.01$ & 0.255 \\
\hline$-1.50 \mathrm{D}$ & $48.21 \pm 4.61$ & $<0.001$ & $54.07 \pm 6.92$ & $<0.001$ & $34.45 \pm 17.94$ & 0.353 \\
\hline $0.00 \mathrm{D}$ & $49.28 \pm 4.54$ & 0.002 & $55.93 \pm 8.27$ & 0.696 & $37.41 \pm 19.77$ & 0.588 \\
\hline$+1.50 \mathrm{D}$ & $50.04 \pm 4.21$ & 0.712 & $56.28 \pm 7.83$ & 0.211 & $34.69 \pm 15.85$ & 0.502 \\
\hline$+3.00 \mathrm{D}$ & $50.59 \pm 4.66$ & 0.127 & $57.22 \pm 8.11$ & 0.031 & $31.90 \pm 13.97$ & 0.106 \\
\hline
\end{tabular}

${ }^{*} p$-Value is calculated by comparing each lens power against the naked eye.

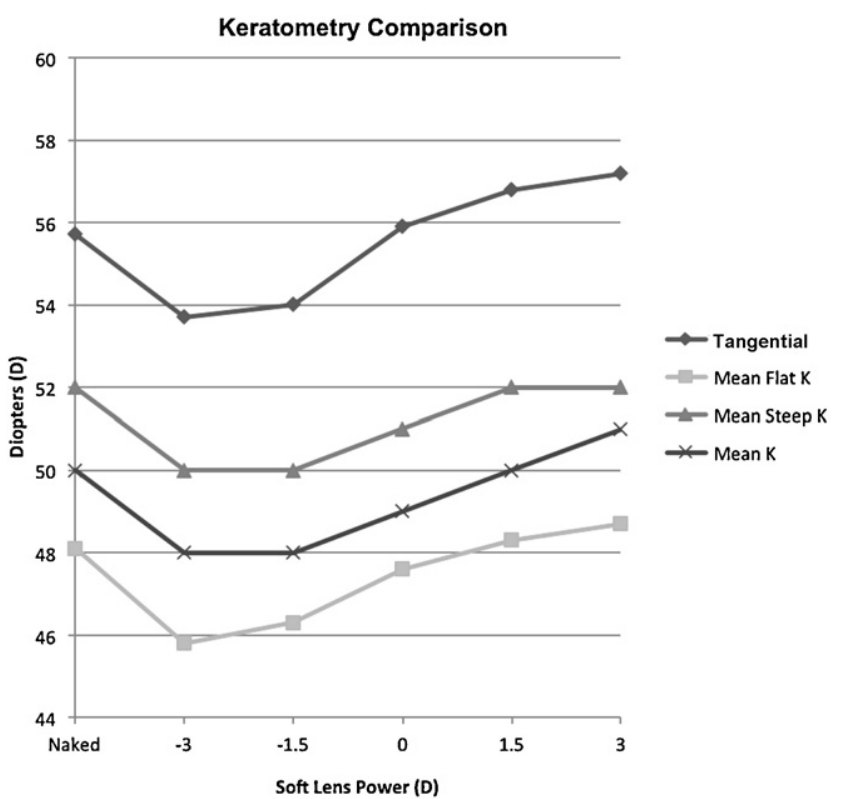

Fig. 2. Mean, flattest and steepest central keratometry and maximum tangential curvature on the naked eye as well as with different soft contact lens powers.

Table 2

Corneal eccentricities at 6 and $8 \mathrm{~mm}$ chord diameters.

\begin{tabular}{|c|c|c|c|c|}
\hline \multirow[t]{2}{*}{ Condition } & \multicolumn{2}{|c|}{$6 \mathrm{~mm}$ diameter } & \multicolumn{2}{|c|}{$8 \mathrm{~mm}$ diameter } \\
\hline & Ecc & $p^{*}$ & Ecc & $p^{*}$ \\
\hline Naked-eye & \multicolumn{2}{|c|}{$0.63 \pm 0.41$} & \multicolumn{2}{|c|}{$0.78 \pm 0.32$} \\
\hline$-3.00 \mathrm{D}$ & $0.64 \pm 0.47$ & 0.799 & $0.89 \pm 0.27$ & 0.001 \\
\hline$-1.50 \mathrm{D}$ & $0.55 \pm 0.40$ & 0.171 & $0.88 \pm 0.26$ & 0.007 \\
\hline $0.00 \mathrm{D}$ & $0.57 \pm 0.39$ & 0.367 & $0.91 \pm 0.25$ & 0.003 \\
\hline$+1.50 \mathrm{D}$ & $0.56 \pm 0.43$ & 0.355 & $0.93 \pm 0.26$ & 0.005 \\
\hline$+3.00 \mathrm{D}$ & $0.56 \pm 0.49$ & 0.488 & $0.95 \pm 0.27$ & 0.004 \\
\hline
\end{tabular}

* $p$-Value is calculated by comparing each lens power against the naked eye. Ecc, eccentricity.

$(p=0.358)$, and in secondary astigmatism aberrations for all lens powers, except with $-3.00 \mathrm{D}$ power lens $(p=0.117)$. The results of the anterior corneal surface aberrations are summarized in Table 3.

\section{Discussion}

The fitting of GP contact lenses is normally the most widely accepted option for managing keratoconus patients, because these lenses can effectively mask corneal irregularity and thus improves visual acuity. However, some patients might not tolerate the discomfort associated to the use of GP lenses. In these cases, piggyback fitting is usually recommended. However, the fitting of GP onto a soft contact lens can be challenging to the eye care practitioner. Such fittings require soft and GP lenses to move independently from each other with blinking and appropriate GP centration over the soft contact lens. Although it is commonly recommended the use of a low-positive powered soft contact lens for piggyback fittings, little is known about the most appropriate soft contact lens power to be employed in these fittings. To the best of our knowledge, this is the first study to assess the most adequate soft contact lens power for piggyback lens fitting. Against commonly accepted practice, the use of a negative-powered soft contact lens provides a better surface for the GP lens to fit over the soft lens. In fact, we found a decrease in corneal keratometry and maximum tangential curvature with negative-powered lens powers, which aids in regularizing the anterior corneal curvature and shape. A flatter surface as a result of fitting a negative-powered soft lens powers over the cornea will require a less negative and thus lighter and more stable GP lens to fit over the soft contact lens. On the contrary, the fitting of a positive-powered soft lens would require a GP contact lens of stronger power.

The elevation topography map has been proposed as the one which more accurately represents the true corneal shape, because it is independent of axis, orientation and position [18]. In our study, we did not find any significant differences in the maximum front elevation of the cornea between the different soft contact lens powers assessed, suggesting that soft contact lens power has little effect over the true anterior corneal shape. The latter is surprising because $\mathrm{a}-3.00 \mathrm{D}$ lens is likely to have differences in thickness across the optic zone of the order of several dozens of microns. Although the Pentacam instrument might not be able to detect such small differences in lens thickness, the fitting of a soft contact lens over the keratoconic eye is expected to regularize areas of lower elevation surrounding the cone. Taking the latter into account, a negative soft lens power may be more suitable than a positive lens for piggyback

Table 3

Anterior corneal surface aberrations for $6.0 \mathrm{~mm}$ pupil diameters.

\begin{tabular}{|c|c|c|c|c|c|c|c|c|}
\hline \multirow[t]{2}{*}{ Condition } & \multicolumn{2}{|c|}{ High order aberrations (RMS) } & \multicolumn{2}{|l|}{ Spherical-like } & \multicolumn{2}{|l|}{ Coma-like } & \multicolumn{2}{|c|}{ Secondary astigmatism } \\
\hline & Value & $p^{*}$ & Value & $p^{*}$ & Value & $p^{*}$ & Value & $p^{*}$ \\
\hline Naked-eye & \multicolumn{2}{|c|}{$2.22 \pm 1.13$} & \multicolumn{2}{|c|}{$0.42 \pm 0.28$} & \multicolumn{2}{|c|}{$1.98 \pm 1.07$} & \multicolumn{2}{|c|}{$0.40 \pm 0.28$} \\
\hline$-3.00 \mathrm{D}$ & $2.02 \pm 0.96$ & 0.012 & $0.40 \pm 0.33$ & 0.642 & $1.70 \pm 1.00$ & 0.001 & $0.49 \pm 0.32$ & 0.117 \\
\hline$-1.50 \mathrm{D}$ & $2.08 \pm 1.06$ & 0.082 & $0.39 \pm 0.22$ & 0.294 & $1.74 \pm 0.94$ & 0.009 & $0.53 \pm 0.41$ & 0.037 \\
\hline $0.00 \mathrm{D}$ & $2.12 \pm 1.19$ & 0.284 & $0.40 \pm 0.31$ & 0.705 & $1.76 \pm 0.91$ & 0.019 & $0.54 \pm 0.37$ & 0.031 \\
\hline$+1.50 \mathrm{D}$ & $2.18 \pm 0.99$ & 0.117 & $0.42 \pm 0.36$ & 0.417 & $1.76 \pm 0.86$ & 0.007 & $0.56 \pm 0.34$ & 0.039 \\
\hline$+3.00 \mathrm{D}$ & $2.21 \pm 0.90$ & 0.854 & $0.45 \pm 0.39$ & 0.888 & $1.85 \pm 0.79$ & 0.358 & $0.60 \pm 0.45$ & 0.007 \\
\hline
\end{tabular}

${ }^{*} p$-Value is calculated by comparing each soft lens power against the naked eye. 
fitting because of its thinner central thickness, which will allow greater corneal oxygenation [12]. In fact, measurements of thickness undertaken in our lab (ET-3, Rehder Development Company, Castro Valley, CA) on a plano-powered Senofilcon A lens revealed a value of $70 \mu \mathrm{m}$ at the center of the lens, with values increasing up to $80 \mu \mathrm{m}$ at $4 \mathrm{~mm}$ chord and decreasing to $15 \mu \mathrm{m}$ at $8 \mathrm{~mm}$ chord. Despite these differences in thickness, it seems that soft contact lens power does not affect anterior shape of the surface resultant from fitting a soft lens over the keratoconic cornea and, consequently, it might not affect GP lens centration.

The eccentricity of the normal cornea normally ranges between 0.35 and 0.45 , but keratoconus disease is associated to greater corneal eccentricity. In our sample, we have found mean corneal eccentricities of 0.63 and 0.78 at $6 \mathrm{~mm}$ and $8 \mathrm{~mm}$ chord diameters, respectively. A previous study found positive-powered soft lenses to improve centration of back-surface spherical GP lens designs fitted over the soft lens in comparison to negative-powered soft lenses [15]. However, since keratoconus disease is associated with increased corneal eccentricity, back-surface spherical GP lenses (eccentricity $=0$ ) might not be the most appropriate GP design for piggyback fitting. Back-surface aspherical GP lenses with eccentricities around 0.8 are more likely to better conform the ecstatic cornea of keratoconus subjects. Alternatively, spherical GP contact lens designs with multiple peripheral curves are also likely to better mimic the corneal shape in keratoconus. In our study, soft lens power had no influence on corneal eccentricity at $6 \mathrm{~mm}$ chord diameter. However, all soft lens powers increased surface eccentricity at $8 \mathrm{~mm}$ of diameter chord, particularly with positivepowered soft lenses. Therefore, negative-powered soft lenses might be better option for piggyback fitting in keratoconus in terms of reducing corneal eccentricity.

In keratoconus, corneal asymmetry and irregularity induce an increase of high-order aberrations, specially coma- and sphericallike aberrations, in comparison to normal corneas [19], and these aberrations together with vertical coma have been proposed as the major aberrations affecting visual quality in keratoconus subjects [20]. In our study, no statistical differences were found in HOA RMS except for $-3.00 \mathrm{D}$ lens power, being the value of the RMS lower with this power. Spherical-like aberration did not change at all, irrespective of the lens power fitted, which is in agreement with a previous study [21]. However, we found that coma-like aberration reduces with soft lenses, more when the power of the lens is more negative, and secondary astigmatism increases for all soft lens powers, except for $-3.00 \mathrm{D}$, being higher when the power of the lens was more positive. So, negative soft lens power seems to be more suitable in terms of anterior corneal aberrations for piggybacking in keratoconus. The results related to corneal aberrations presented in this study should be interpreted with caution as a previous study reported questionable repeatability on measuring corneal wavefront aberrations with the Pentacam instrument [22]. Furthermore, another study reported Zernike coefficients measured by the Pentacam device to be higher than those provided by other measuring devices [19]. Nevertheless, it is of interest the reduction in coma-like aberrations with negative soft lens powers found in this study as coma-like coefficients measured by Pentacam have been reported to be more accurate than other coefficients measured with the same device [23].

A limitation of this study was the theoretical approach undertaken to assess the most appropriate soft contact lens power for piggyback fitting as we did not fit GP lenses over the different soft lenses employed in this study. Therefore, the findings of this study need to be confirmed with a clinical study in which different GP designs are fitted over different soft contact lens powers in keratoconus subjects. And, in addition to assessing surface curvature and aberrations, it would be also desirable to assess lens centration, movement and comfort.

In summary, the results of this study show that negativepowered soft contact lenses are likely to be more suitable for piggyback fitting than positive-powered lenses in subjects with keratoconus.

\section{Conflict of interest}

Jacinto Santodomingo-Rubido is a full-time employee of Menicon Co., Ltd. Miguel Romero-Jiménez is undertaking a PhD partly funded by Menicon Co., Ltd. at the Clinical \& Experimental Optometry Research Lab, Center of Physics, University of Minho, Braga, Portugal.

\section{References}

[1] Rabinowitz YS. Keratoconus. Survey of Ophthalmology 1998;42:297-319.

[2] Romero-Jiménez M, Santodomingo-Rubido J, Wolffsohn J. Keratoconus: a review. Contact Lens Ant Eye 2010;33:157-66.

[3] Romero-Jiménez M, Santodomingo-Rubido J, González-Meíjome JM. The thinnest, steepest and maximum elevation locations in non-contact and contact lens wearers in keratoconus. Cornea 2012 [Epub ahead of print].

[4] Edwards M, McGhee CN, Dean S. The genetics of keratoconus. Clinical and Experimental Ophthalmology 2001;29:345-51.

[5] McMahon TT, Szczotka-Flynn L, Barr JT, Anderson RJ, Slaughter ME, Lass JH et al. A new method for grading the severity of keratoconus: the keratoconus severity score (KSS). Cornea 2006;25:794-9.

[6] Colin J, Malet FJ. Intacts for the correction of keratoconus: two-year follow-up. Journal of Cataract and Refractive Surgery 2007;33:69-74.

[7] Caporossi A, Mazzotta C, Baiocchi S, Caporossi T. Long-term results of rivoflavin ultraviolet a corneal collagen cross-linking for keratoconus in Italy: the Siena eye cross study. American Journal of Ophthalmology 2010;149:585-93.

[8] Baldone JA, Clark WB. Contact lenses in the aphakic child. Contact Lens Medical Bulletin 1970;3:25.

[9] Baldone JA. The fitting of hard lenses onto soft contact lenses in certain diseased conditions. Contact Lens Medical Bulletin 1973;6:15.

[10] Westerhout D. The combination lens and therapeutic uses of soft lenses. Contact Lens 1973;4:3-9.

[11] Kok JHC, van Mil C. Piggyback lenses in keratoconus. Cornea 1993;12:60-4.

[12] Lopez-Alemany A, Gonzalez-Meijome JM, Almeida JB, Parafita MA, Refojo MF. Oxygen transmissibility of piggyback systems with conventional soft and silicone hydrogel contact lenses. Cornea 2006;25:214-9.

[13] Florkey LN, Fink BA, Mitchell GL, Hill RM. Corneal oxygen uptake with piggyback contact lens systems. Cornea 2007;26:324-35.

[14] Baldone JA. Piggy-Back fitting of contact lenses. CLAO Journal 1985;11:130-4.

[15] O'Donnell C, Codina CM. A hyper-Dk contact lens system for keratoconus. Eye Contact Lens 2004;30:44-8.

[16] Sengor T, Kurna SA, Aki S, Özkurt Y. High Dk piggyback system for contact lens-intolerant keratoconus patients. Clinical Ophthalmology 2011;5:331-5.

[17] Lebow KA, Grohe RM. Differentiating contact lens induced warpage from true keratoconus using corneal topography. CLAO Journal 1999;25:114-22.

[18] Miháltz K, Kovács I, Takács A, Nagy ZZ. Evaluation of keratometric, pachymetric, and elevation parameters of keratoconic corneas with Pentacam. Cornea 2009;28:976-80.

[19] Piñero DP, Alió JL, Alesón A, Escaf M, Miranda M. Pentacam posterior and anterior corneal aberrations in normal and keratoconic eyes. Clinical and Experimental Optometry 2009;92:297-303.

[20] Okamoto C, Okamoto F, Oshika T, Miyata K, Samejima T. Higher-order wavefront aberration and letter-contrast sensitivity in keratoconus. Eye 2008;22:1488-92.

[21] Marsack JD, Parker KE, Niu Y, Pesudovs K, Applegate RA. On-eye performance of custom wavefront-guided soft contact lenses in a habitual soft lens-wearing keratoconic patient. Journal of Refractive Surgery 2007;23:960-4.

[22] Shankar H, Taranath D, Santhirathelagan CT, Pesudovs K. Repeatability of corneal first-surface wavefront aberrations measured with Pentacam corneal topography. Journal of Cataract and Refractive Surgery 2008;34:727-34.

[23] Piñero DP, Saenz C, Alió JL. Intraobserver and interobserver repeatability of curvature and aberrometric measurements of the posterior corneal surface in normal eyes using the Pentacam system. Journal of Cataract and Refractive Surgery 2009;35:113-20 http://www.ncbi.nlm.nih.gov/pubmed/18041254 